\title{
Excavaciones arqueológicas en el yacimiento altomedieval de Revenga: Nuevos datos para el conocimiento de los espacios de hábitat altomedieval en el Alto Arlanza (Burgos)
}

Karen Álvaro Rueda, Esther Travé Allepuz, M. Dolores López Pérez Universidad de Barcelona

Early medieval habitat, worship and production structures at Revenga (Comunero de Revenga, Burgos): Archaeological evidence for a new historical approach 
Resumen: Las dificultades inherentes al análisis de las estructuras de poblamiento y organización del territorio en el periodo altomedieval se deben en buena medida a la escasez de fuentes para su conocimiento. Los trabajos de excavación arqueológica en el yacimiento de Revenga (Comunero de Revenga, Burgos), han permitido conocer los rasgos característicos del espacio de hábitat anexo a una de las necrópolis más conocidas del Alto Arlanza y han aportado información muy valiosa para el

Abstract: The Upper Arlanza Basin is part of a complex scenario. The archaeological site of Revenga (Comunero de Revenga, Burgos) is one of the rock-cut necropolises existing between the headwaters of rivers Arlanza and Duero. These sites are identified by the archaeological remains of a church, a cemetery of rock-cut tombs, and a settlement in the annexe area. The inhabitation areas of the landscape at the Upper Arlanza Basin have been known for the first time since the archaeological fieldwork started in 2014 at the archaeological site of Revenga. The main features of settlements at the Upper Arlanza Basin seem to relate to the association between cemeteries, churches and habitat -inecclesiamento, according to M. Lauwers $(2005,2013)$. This would have settled a network that cannot be related with a clear and systematic organization or with firm and well-settled power structures at least during the earlier period.

The study of the vast 800 -square-metre-wide area at the north-east of the necropolis and the archaeological excavation carried out during 2014 and 2015 revealed the existence of a considerable amount of habitat units, generally identified as sunken-featured buildings. Also few production areas exploited in at least three different phases were discovered. These remains prove the existence of an intensely occupied space during an extended period of time. In this paper we will introduce a brief summary of results obtained until the present day, their implications for the historical narrative and the new work hypotheses that can be formulated.

Recent fieldwork at the site of Revenga revealed the existence of such architecture, from which only the footprint on the rocky surface has been preserved. A considerable amount of oval and squared mostly sunken-featured buildings have been found out at the site of Revenga, occasionally with associated sediment. Some of them are related to use and abandonment layers, others appeared just after the rock surface cleaning and conocimiento de estas comunidades. En este trabajo se presentan los resultados de las primeras intervenciones realizadas y se interpretan las implicaciones históricas de dichos datos, que permiten adelantar al menos dos siglos las cronologías tradicionalmente atribuidas a estos enclaves.

Palabras Clave: Poblamiento, arquitectura rupestre, territorio, paisaje, Alto Arlanza finally some others were identified during the lab phase, when studying the site outline and the aerial photography.

The entire assemblage exhibits similar and quite homogeneous features. These structures are cut on the floor surface according to an oval or squared plan. They are between 5 and $10-\mathrm{m}^{2}-$ wide and occasionally larger, reaching about $15 \mathrm{~m}^{2}$ wide, even though these larger structures do not transform significantly the overview of the site. This panorama does not seem to be really different than protohistoric habitat found in mountain areas of the Cantabrian range (Ruano, 2015). The assemblage of data gathered at the archaeological site strengthens the hypothesis of a long-lasting settlement at the site of Revenga in accordance with different phases. These imply the transformation and rebuilding of habitat and worship structures. The relative chronology of layers defines at least two different phases of settlement: one of structures predominantly oval and a later one of squared structures.

Main volumes of ceramic sherds, generally scarce, can be attributed to the Late Antique period, potentially to the 4th 6th Centuries, which stands for the occupation of these sites earlier than assumed. Data gathered from the archaeological approach in recent campaigns suggest that the occupation of Revenga might have taken place at least a couple of centuries earlier than considered in previous research (Padilla \& Álvaro, 2010: 293). Habitat structures and open-air production areas would have been occupied from the 7th Century onwards, according to similar chronologies suggested at some other similar sites (Vigil-Escalera, 2007). Therefore, the panorama is that of settlement patterns still forming in early medieval times the origin of which has its roots in the late antique period.

KeYwords: Settlement, rock-cut buildings, territory, landscape, Upper Arlanza Basin 


\section{INTRODUCCIÓN}

El territorio del Alto Arlanza es bien conocido por el extraordinario conjunto de necrópolis rupestres diseminadas en el corredor que discurre entre las cabeceras de los ríos Arlanza y Duero, en la alta sierra pinariega (Fig. I). Superados los trabajos iniciales de A. del Castillo en dichas necrópolis por tierras burgalesas y sorianas, el estudio posterior de estos asentamientos ha buscado superar la parcialidad y fragmentación del registro arqueológico, que viene condicionada por el hecho de que sólo conocemos los espacios funerarios de estos lugares y sabemos muy poco o nada del poblamiento (Padilla y Álvaro, 20IO: 26I), es decir de sus estructuras de hábitat.

Un análisis pormenorizado de los restos visibles en clave territorial (Álvaro, 20I2) permite observar una compleja estructuración del espacio en base a una densa red de poblamiento en donde se advierten unidades de hábitat mayores, como Revenga o $\mathrm{Cu}$ yacabras, junto con otros asentamientos menores y entidades nucleares (Padilla y Álvaro, 20I0: 292). La información histórica que tenemos de esta área es muy escasa para el periodo anterior al siglo XI, cuando la cuenca alta del río Arlanza es mencionada en las fuentes por primera vez. La documentación nos habla del proceso de formación del paisaje y la organización territorial del sistema feudal (Pastor, 1996: 48-55), pero no proporciona información relativa al origen de las comunidades que lo habitan.
En esta zona de montaña, la más alta y menos romanizada de los territorios circundantes, la altitud -entre los IOOO y $1200 \mathrm{~m}$ - ha condicionado históricamente las condiciones de vida de sus habitantes, y confiere una serie de rasgos propios al conjunto de asentamientos. Precisamente la altitud de estos establecimientos, unida al clima relativamente extremo del entorno serrano, contribuye al aislamiento de las comunidades que habitan este espacio, que queda relegado como entidad periférica. La alta sierra se mantiene al margen de las estructuras territoriales existentes en época romana, cuyos vestigios se documentan a cotas más bajas, a partir de las localidades de Palacios de la Sierra y Salas de los Infantes.

El asentamiento de Revenga pertenece al Comunero de Revenga, que lo conforman los municipios de Quintanar, Canicosa y Regumiel de la Sierra (Burgos) y que lo administran de forma mancomunada. La necrópolis rupestre se alza sobre un promontorio rocoso (Padilla y Álvaro, 20I3) que preside una amplia explanada de prado y bosque donde se observan numerosos indicios de las estructuras de hábitat. Las primeras investigaciones llevadas a cabo, que tradicionalmente se habían dirigido al estudio de los cementerios de tumbas excavadas en la roca y sus centros de culto, descuidaron en buena medida la observación de los espacios domésticos anexos a estos recintos funerarios. En el área contigua a la necrópolis de Revenga, el suelo rocoso ofrece numerosos elementos cuya lectura proporciona información muy valiosa para el estudio de las formas de vida de 


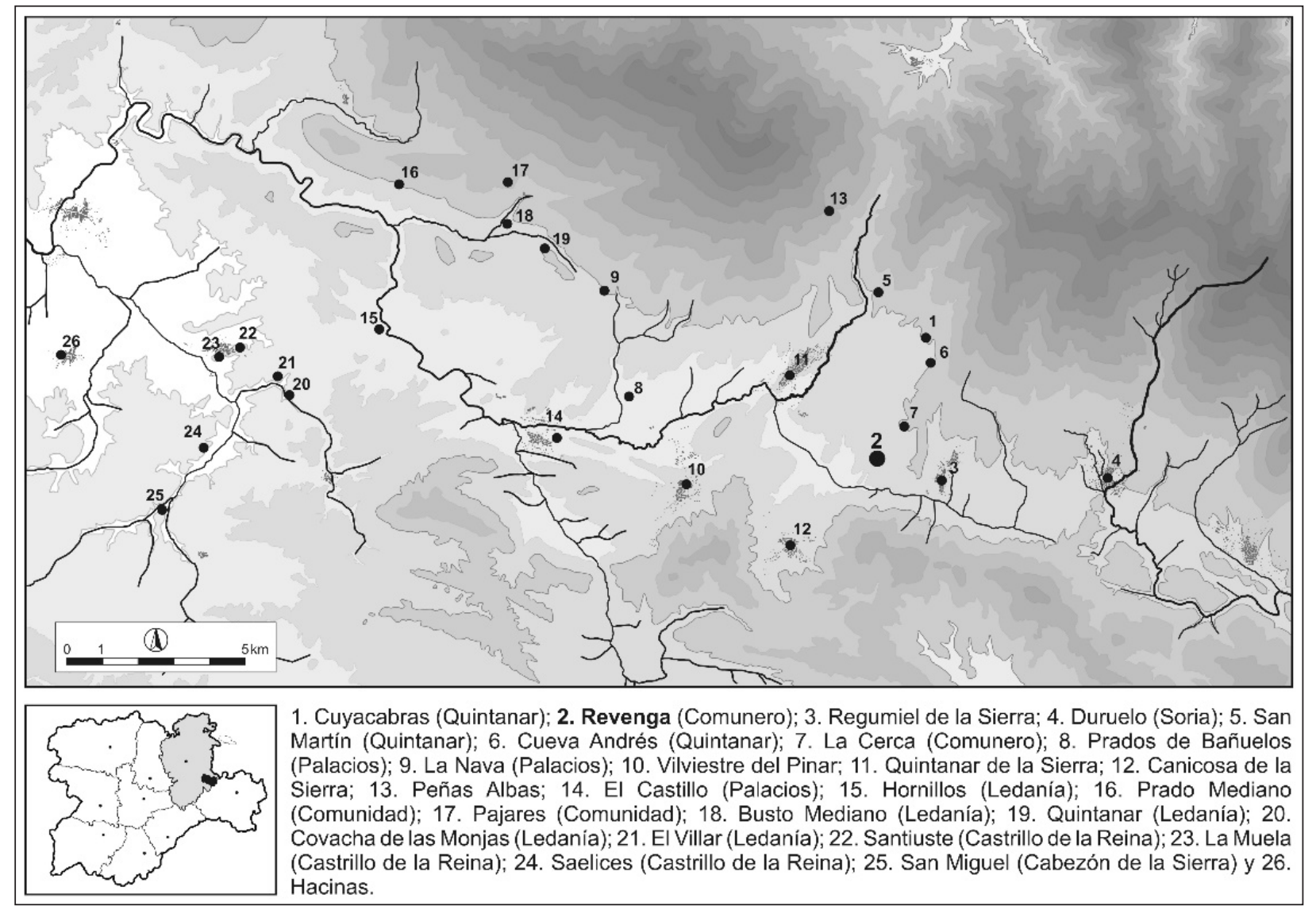

Figura I: Mapa de localización de las necrópolis rupestres en la cuenca alta del río Arlanza, entre las que se cuenta el despoblado de Revenga.

estas comunidades altomedievales. Por primera vez, hemos tenido ocasión de aproximarnos a estas áreas de poblamiento gracias a los trabajos de excavación que desde 2014 realizamos en el yacimiento de Revenga, cuyos resultados preliminares ofrecemos a lo largo de este trabajo.

El estudio del terreno situado al nordeste de la necrópolis conocida, en una extensión que supera los 800 $\mathrm{m}^{2}$ intervenidos, nos ha permitido advertir la existencia de numerosos fondos de cabaña, y algunas áreas de producción, que pueden articularse como mínimo en tres fases sucesivas y que revelan la existencia de un espacio intensamente ocupado durante un periodo de tiempo prolongado. En las páginas que siguen presentamos un análisis sumario de los resultados obtenidos hasta ahora, de las implicaciones historiográficas de la interpretación del registro arqueológico y de las necesidades que de ello se derivan a la hora de plantear la investigación futura.

Nuestra impresión general e hipótesis de trabajo es que el Alto Arlanza habría constituido un espacio marginal en relación con los centros de poder tardorromanos y visigodos durante la transición entre la antigüedad tardía y el mundo feudal, y que como tal se mantiene al margen de la organización progresiva del condado de Castilla y, en concreto, de la articulación del territorio que acabará constituyendo el Alfoz de Lara hasta su inclusión en el mismo en el siglo XI (Escalona, 2002). El espacio, a juzgar por las características del poblamiento en Revenga que comentaremos a lo largo de este 
trabajo, parece estar habitado en época temprana por unas comunidades autóctonas que no muestran visos de romanización, y que se ven inmersas en el proceso paulatino de arraigo de las prácticas cristianas. En este sentido, las primeras estrategias de organización del espacio llegaron con posterioridad, motivadas por la actividad eclesiástica de evangelización del territorio, en un proceso de construcción del paisaje cristiano no necesariamente relacionado con las transformaciones feudales de finales del siglo X sino con un mundo de microcristiandades (García de Cortázar 2012: I46) que se configura con anterioridad.

\section{IGLESIAS, NECRÓPOLIS Y ESPACIOS DE HÁBITAT}

El análisis del poblamiento en Revenga no puede desvincularse de las características de los yacimientos del Alto Arlanza. Todos ellos vinculan un centro de culto, una necrópolis y un área de hábitat más o menos extensa. Los indicios que han permitido localizar este poblamiento en Revenga se advierten del mismo modo en otros yacimientos de la zona. La ocupación y evolución de todos estos enclaves parecen responder a un proceso de asociación de cementerios, iglesias y poblamiento -inecclesiamento, en palabras de M. Lauwers (2005, 2013) - que habría sido el resultado de la confluencia de dos comportamientos sociales paralelos: por un lado, la edificación de lugares de culto de los propietarios rurales en el seno de sus tierras (Sotomayor 2004: 529), y por otro la búsqueda por parte de una sociedad eminentemente cristiana de un espacio de inhumación ad sanctos (Duval, 1988). El fenómeno de fundación y consagración de estas iglesias rurales ya está sólidamente implantado en el siglo IV (Ripoll y Velázquez, 1999: I07). A esta red pertenecerían buena parte de los edificios rurales que pone al descubierto la arqueología, a pesar de que en este momento aún no puede relacionarse el conjunto de pequeñas iglesias rurales con una organización clara ni sistemática ni con unas estructuras de poder eclesiástico firmes.

Tal vez las iglesias de las comunidades de Revenga y $\mathrm{Cu}-$ yacabras (Álvaro y Padilla, 2012) constituyan un ejemplo de la materialización sobre el paisaje de este cristianismo primitivo, aglutinando a su alrededor un poblamiento que empieza a ser concentrado. Aun así, no será hasta más tarde cuando estos lugares se constituyan como nodos articuladores del territorio, tanto por su papel de liderazgo espiritual y político, como económico y mental, en cuanto a su alrededor se sitúan los espacios agrícolas, símbolo de estabilidad y orden en contraste con el espacio boscoso de las periferias (García de Cortázar, 20I2: I64). El carácter marginal de Revenga permitirá a esta comunidad mantenerse de forma más o menos autónoma, con un centro neurálgico que es la iglesia y que ejerce como elemento de cohesión de los habitantes.

Tanto la documentación escrita como las evidencias arqueológicas permiten situar el abandono de estos centros - una vez constituida la red parroquial de época feudal-hacia finales del siglo XIII, pero no conocemos prácticamente nada acerca del origen del poblamiento en el Alto Arlanza ni, en particular, de las formas de hábitat, producción y explotación de los recursos. Las causas de este vacío son múltiples, pero, entre ellas, la monumentalidad que reviste el espacio funerario acaba convirtiendo el centro de culto y la necrópolis en foco de atención por encima de los elementos de habitación. La preeminencia del cementerio junto con la invisibilidad de las estructuras de hábitat termina por limitar el conocimiento de los poblados y no permite reconocer más que un conjunto de necrópolis y ermitas (Quirós, 2006: 65). Sin embargo, la dificultad para identificar estos asentamientos también viene dada por la propia naturaleza de los mismos. El uso extendido de la madera como material de construcción básico para este periodo (Wickham, 2005: 486, 49I), probablemente en relación con la necesidad del campesinado de construir sus viviendas sin mano de obra especializada, y su carácter perecedero condiciona en buena medida la desaparición de estas estructuras dejando únicamente su huella sobre el terreno en el mejor de los casos.

No parece discutible que las ausencias notables que plantea el registro arqueológico hacen necesaria la intervención en los espacios anexos a estos cementerios. En esta línea se ha desarrollado recientemente un proyecto de investigación que aspira a tener continuidad 


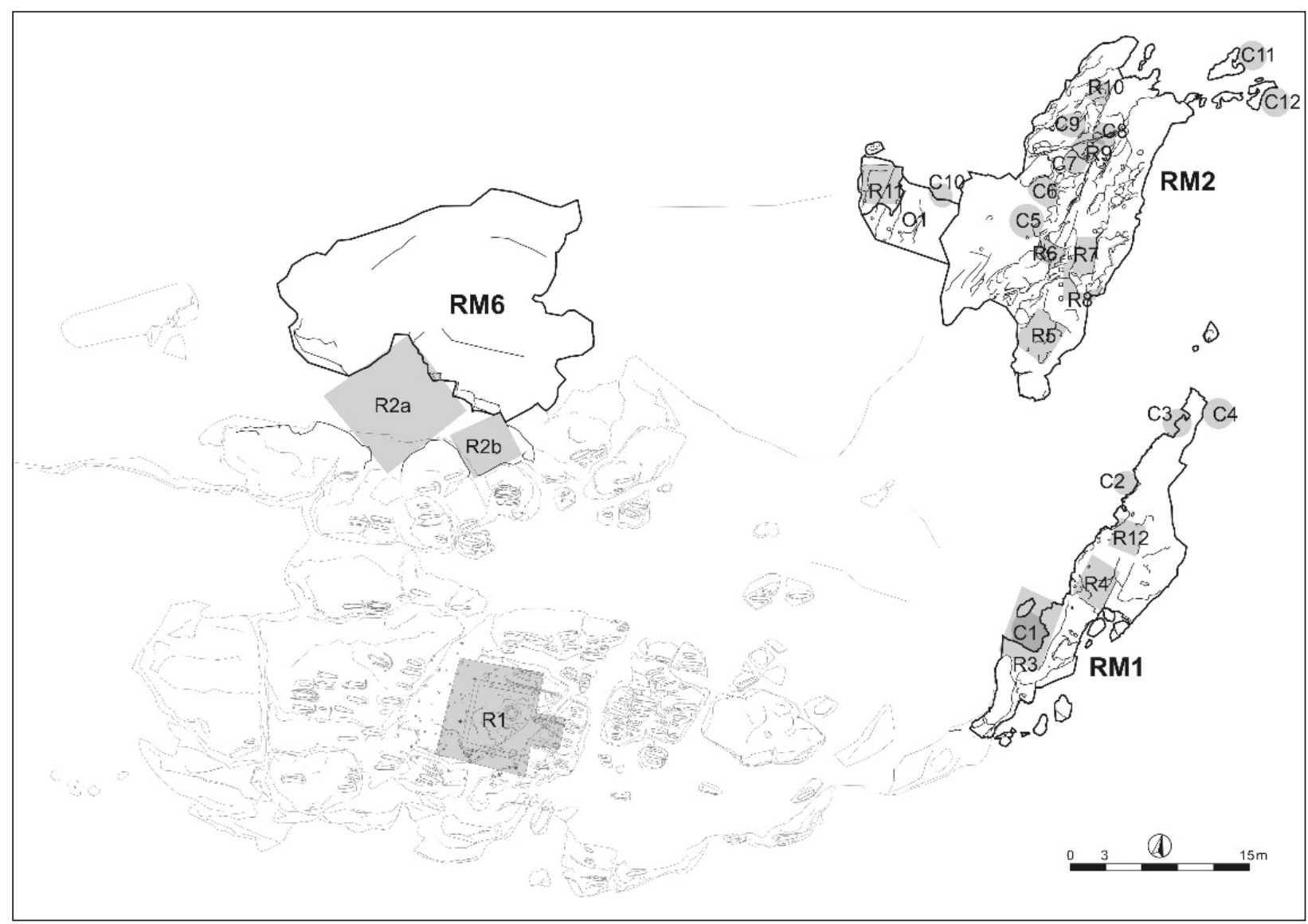

Figura 2: Planta general de la necrópolis de Revenga y sus inmediaciones, con indicación de las principales estructuras detectadas sobre el suelo rocoso de las floraciones RMi, RM2 y RM6.

en los años venideros y que ha permitido llevar a cabo las labores arqueológicas en el yacimiento de Revenga. El objetivo, sin duda, pasa por actualizar un registro deficiente a fin de llevar a cabo el análisis de las comunidades rurales y su estructura tal como ya reclamó desde hace tiempo la comunidad científica (Escalona, 2002: 63-64; Quirós, 2006). Los trabajos llevados a cabo desde entonces han puesto en evidencia la existencia de un poblamiento aglomerado, cuyo análisis e interpretación requiere de estudios analíticos con un sólido planteamiento de base y de aproximaciones integrales para su interpretación (Quirós, 20I4: I44).

El planteamiento de una excavación en extensión y la lectura detallada de los vestigios que este espacio nos ofrece nos pone en condiciones de plantear una primera interpretación acerca del hábitat, su estructuración y características en este emblemático yacimiento (Fig. 2). La realización de estos trabajos ha revelado la existencia de una estratigrafía de poca potencia, pero gran complejidad de relación, que pone al descubierto las trazas habitualmente en forma de huellas negativas de un espacio muy arrasado con reutilizaciones frecuentes.

Una intervención intensiva y en extensión nos ha permitido definir una secuencia estratigráfica que agota el sedimento y cuya lectura permite identificar la existencia de distintas estructuras domésticas o fondos de cabaña ${ }^{1}$, así como algunas áreas de producción. Estos

\footnotetext{
${ }^{1}$ La terminología fondo de cabaña, literalmente adoptada del francés fond de cabanne (Chapelot, 1980), ha sido en ocasiones cuestionada, dado que el
} 


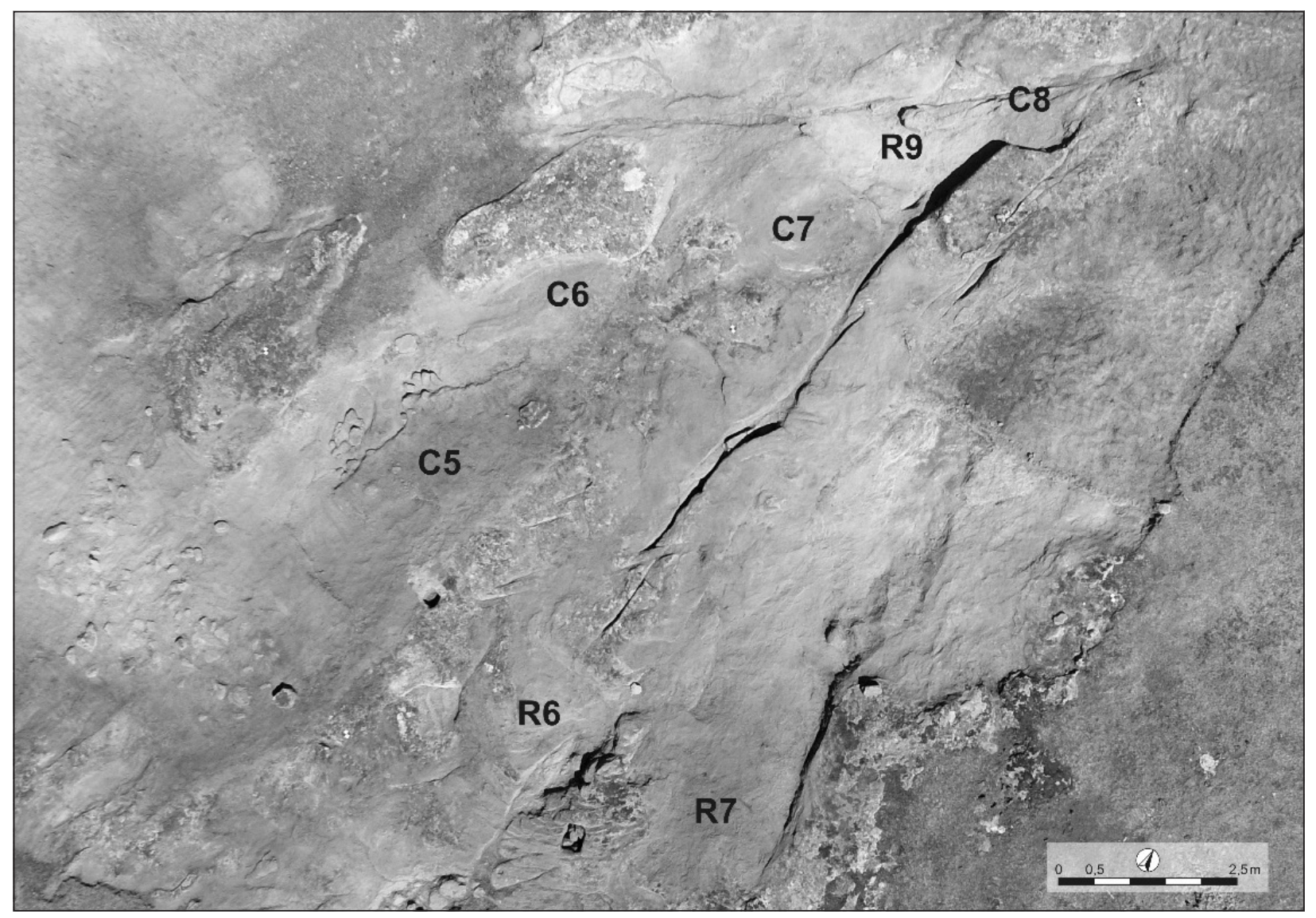

Figura 3: Fotografía aérea del suelo rocoso RM2 con identificación de las estructuras visibles.

elementos reflejan una notable variabilidad tipológica por lo que nos referiremos a ellos de manera genérica empleando el término de estructuras de hábitat o de culto, siendo conscientes que estamos interpretando una funcionalidad de las mismas y que se debe analizar la morfología precisa en cada caso.

concepto cabaña, especialmente en contextos altomedievales, denota una idea de pobreza, tosquedad, marginalidad o provisionalidad, además de las implicaciones de dicho vocablo en relación con la presunción de funcionalidad al margen de la caracterización arqueológica y formal (Azkárate y Quirós, 200I: 28). Investigaciones recientes (Tejerizo 20I4: 217) han optado preferentemente por la terminología anglosajona que se refiere a estos vestigios arqueológicos como estructuras de fondo rehundido en base a la propuesta de P. Rahtz (1976: 70-73), que se basa directamente en el identificativo alemán de las grubenhaüser (West, I969: 4; Addyman y Leigh, 1973: 7 y ss.). La naturaleza dispar de las estructuras recuperadas en Revenga requieren de una terminología suficientemente amplia y genérica como para poder incluir la gran multiplicidad de formas, que no se limitan a una planta rehundida, sino que en ocasiones presenta cimientos realzados sobre una elevación natural del suelo rocoso.
Contamos además con determinados espacios en los que se puede identificar la existencia de una actividad productiva de cualquier tipo y que no necesariamente aparece directamente relacionada con estructuras construidas en materiales perecederos a partir de su cimentación sobre la roca, sino que se trata más bien de ubicaciones al aire libre, por lo que no parece descabellado a priori relacionar los fondos de cabańa con usos domésticos, con sus matices y connotaciones.

Los trabajos de campo recientes en el yacimiento de Revenga han dado a conocer la existencia de este tipo de arquitectura, de la cual sólo se conservan sus huellas sobre la roca. En la explanada de prado y bosque que se extiende al nordeste y sur de la necrópolis respectivamente, se aprecian en el suelo pequeñas áreas donde aflora la roca viva. Estas áreas de arenisca a la vista han 
sido identificadas (RMI-RM8) y a partir de ellas se ha iniciado el proceso de excavación, limpiando su superficie y retirando la cubierta vegetal que se les adosa, dejando al descubierto, en ocasiones algo de estratigrafía y en otros casos directamente la superficie de la roca madre. Los trabajos de movimiento de tierras de las campańas recientes se han concentrado sobre los espacios definidos como RMi y RM2 (Fig. 2) realizando también un sondeo en profundidad en un espacio alejado de la necrópolis $\left(\mathrm{RM}_{4}\right)$ donde ha sido posible recuperar unos $40 \mathrm{~cm}$ de potencia estratigráfica bajo un derrumbe relativamente potente. La identificación y topografía exhaustiva de toda la estratigrafía negativa de estos sectores, junto con la realización de fotografías aéreas desde una altura de $30 \mathrm{~m}$ (Fig. 3), ha permitido identificar el conjunto de estructuras y analizar su morfología y secuencia cronológica.

\section{Caracterización de la ARQuitectura ALTOMEDIEVAL EN REVENGa}

Durante las dos campañas iniciales se han documentado numerosos restos de probables fondos de cabaña de formas circulares y rectangulares, en su mayoría rehundidos, tallados sobre la roca, en casos esporádicos de suelo realzado y en numerosas ocasiones con estratigrafía asociada. Una buena parte de ellos presentan sedimento, mientras que otros carecen de él quedando al descubierto tras una limpieza superficial del suelo. El conjunto de estructuras presenta características bastante homogéneas. Se trata de recortes y rebajes del suelo de planta circular o cuadrada con dimensiones que oscilan entre los 5 y los Io $\mathrm{m}^{2}$ de extensión. En algunos casos, se advierte la presencia de plantas ligeramente superiores, que pueden superar los $15 \mathrm{~m}^{2} \mathrm{de}$ extensión, pero que no parecen transformar la imagen general que advertimos en la organización del hábitat en este espacio, formada por pequeñas cabañuelas o viviendas de reducidas dimensiones. La imagen que se desprende de estas estructuras no difiere excesivamente de las formas de poblamiento protohistórico o castreño documentadas en la cordillera cantábrica (Ruano,
20I5). Sin embargo debemos notar la presencia de dos edificios singulares que no se ajustan a esta caracterización inicial del conjunto: Se trata de dos estructuras de planta cuadrangular (RI y R2) que morfológicamente presentan rasgos muy dispares respecto del conjunto de estructuras. En primer lugar, la estructura Ri manifiesta una funcionalidad religiosa evidente, ya que corresponde con el centro de culto que articula la necrópolis.

La iglesia de Revenga (RI), que conocemos únicamente a partir de sus cimientos tallados sobre la arenisca del lugar, preside la necrópolis que acoge el centenar y medio de sepulturas rupestres documentadas hasta el momento. Las abundantes trazas de esta construcción permiten ofrecer las características generales de la misma, que han sido ampliamente desarrolladas en trabajos anteriores (Padilla y Álvaro, 20IIa: 442-444; 20I3: 18-26; Álvaro y Padilla, 20I2: 450-452). Se trata de un edificio de planta cuadrada, presidido por un ábside rectangular al que se adosa una nave de unos $5 \mathrm{~m} \mathrm{de}$ lado a la cual se accede por una única puerta abierta en el muro de mediodía. El cuerpo principal del edificio contó con un atrio o galería porticada que rodeaba el conjunto por las paredes de poniente y mediodía. Este edificio fue ampliado en una segunda fase, llegando a duplicar la superficie útil aunque conservando la apariencia original. La nueva construcción, pudo contar con una cabecera rectangular más amplia y una planta de nuevo cuadrangular de unos $7 \mathrm{~m}$ de lado, manteniendo el acceso en su ubicación original, en la fachada sur.

Cabe destacar dos elementos singulares relacionados con esta construcción: el primero de ellos es una pileta circular (Padilla y Álvaro, 20Irb; López et al., 2016: 98III) tallada sobre el suelo rocoso, situada en el centro del edificio moderno y probablemente relacionada con esta fase de remodelación y ampliación. El segundo elemento a destacar se trata de un conjunto de enigmáticas insculturas labradas sobre el suelo rocoso en su mayoría bajo el pórtico, en el área de acceso al templo, con motivos de gran carga simbólica que aluden a figuras geométricas, antropomorfas, y zoomorfas ( $\mathrm{Pa}$ dilla y Álvaro, 20Ira: 444-454). Los usos religiosos de este edificio son evidentes y presentan abundantes pa- 


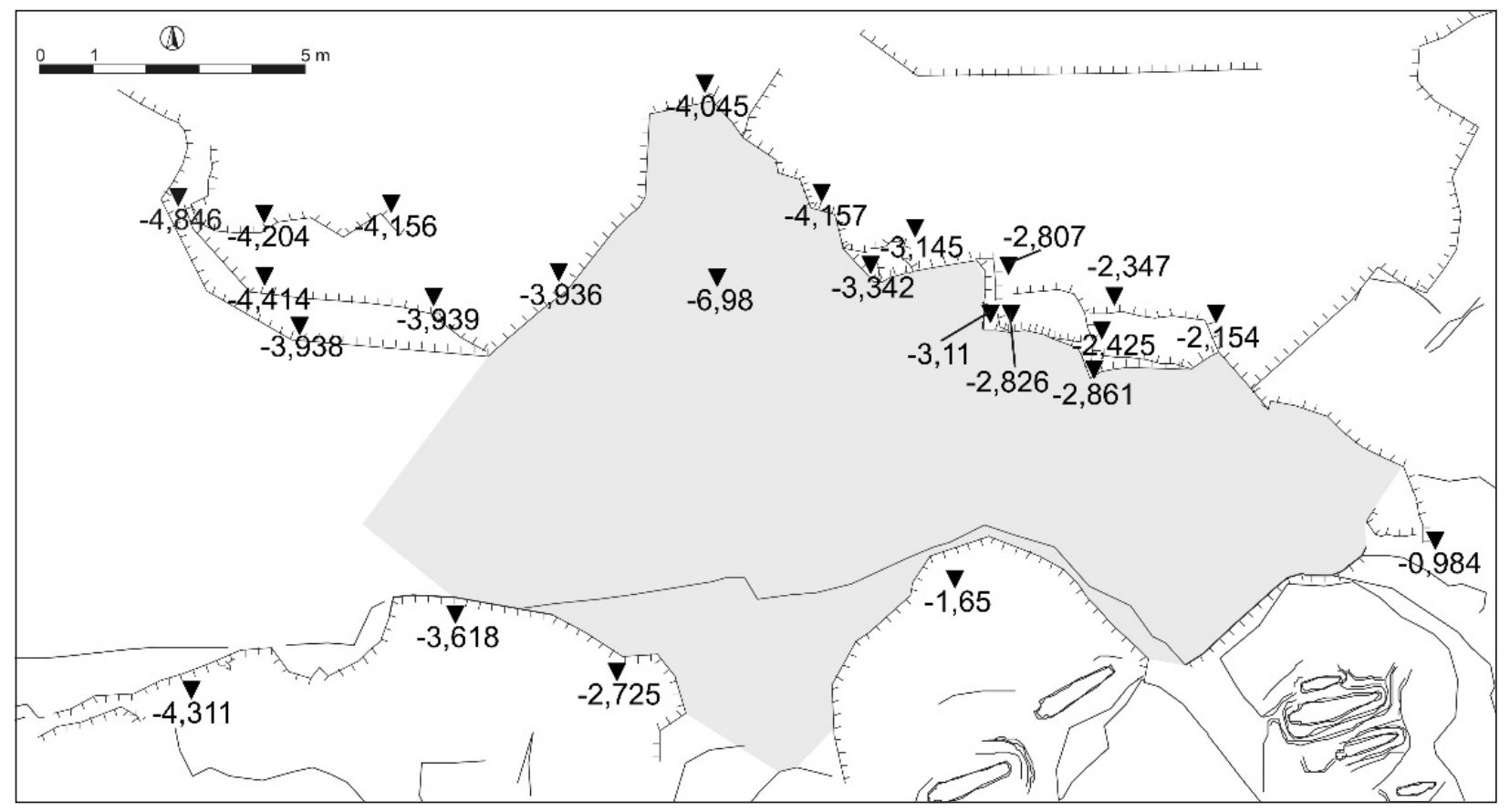

Figura 4: Planta acotada de las estructuras cuadrangulares R2a y R2b.

ralelos en toda la zona, siendo el despoblado de $\mathrm{Cu}-$ yacabras el más significativo (Padilla y Álvaro, 2008: 594-597). De hecho, todas los conjuntos funerarios de la zona se relacionan con un edificio de culto que agrupa a su alrededor las inhumaciones dando origen al recinto funerario (Álvaro y Padilla, 20I2: 449).

Sin embargo, mucho más compleja resulta la interpretación de la enigmática estancia (R2) que se advierte en el límite septentrional de la necrópolis, a la que por ahora no podemos atribuirle funciones precisas en ningún sentido. Dicho espacio, que no ha sido aún excavado constituye el objetivo primordial de las intervenciones futuras. Una prospección superficial del sector y una limpieza no intrusiva de la superficie rocosa a la vista permitieron realizar con precisión la topografía el recinto visible y completar así la planta general de la necrópolis realizada en 1996 (Álvaro, 20I2: 367-370). Esta estructura rupestre implica la talla a plomo de la arenisca hasta configurar paredes cuya profundidad aún desconocemos (Fig. 4). El detalle de una secuencia de pequeños encajes rectangulares correlativos a modo de peldaños, recortados en una diferencia media de cota de unos $20 \mathrm{~cm}$, permiten interpretar la existencia de una posible escalera que salvaría el nivel existente entre el espacio que constituye un primer ámbito de uso (R2a) y la superficie del montículo rocoso en donde se advierte un segundo espacio de planta cuadrada (R2b). En las paredes laterales del sector, se observan también múltiples encajes y recortes que deberán ser objeto de un análisis en detalle. Este recinto deberá ser excavado en su totalidad, ya que en la actualidad está totalmente cubierto por la vegetación que protege un volumen de sedimento que parece considerablemente mayor que la tónica habitual del yacimiento, a juzgar por los derrumbes que afloran en esta zona. Sólo entonces será posible dilucidar la posible función de esta área de dimensiones considerables.

A excepción de estos dos espacios singulares, el conjunto de estructuras en general está formado por habitaciones de dimensiones significativamente más reducidas, ubicadas en un radio de entre 30 y $50 \mathrm{~m}$ 




Figura 5: Planta de detalle del suelo rocoso RM2 con identificación de las estructuras talladas en la roca y los niveles de pavimentación, uso y amortización recuperados. 
al nordeste de la necrópolis. La excavación a partir de la floración de arenisca delimitada como RM2 ha permitido recuperar buena parte de estas estructuras, ocasionalmente con estratigrafía asociada (Fig. 5). Por regla general, los niveles de sedimento en relación con las construcciones identificadas sobre el suelo son escasos, cuando no inexistentes, y sus trazas muy tenues, pero en aquellos casos en donde ha sido posible recuperar algo de estratigrafía, la disposición de la misma parece análoga en la mayoría de casos: Es habitual que la planta del habitáculo se talle en el suelo rocoso mediante un rebaje del mismo, aunque no es extraño que se utilicen espacios llanos tallados someramente por los laterales para constituir una estructura de fondo realzado.

Siendo los fondos rehundidos los más frecuentes, la mayoría presentan pequeñas marcas de talla circulares sobre su superficie probablemente realizadas mediante un punzón romo. Este picoteado del suelo proporciona una superficie rugosa sobre la que asentar una fina capa de pequeños cantos rodados, a veces con algo de arena o gravillas, que constituyen una preparación muy tosca de un pavimento. Esta capa aparece sistemáticamente cubierta por un suelo de tierra batida, liso y bien nivelado, ocasionalmente con algunos carboncillos y algo de cerámica. Entre la preparación de cantos y el suelo de uso arcilloso, en algunos casos se ha podido documentar una segunda capa de preparación formada por un sedimento arenoso o de gravilla muy compactado. Estos niveles de uso, aparecen habitualmente cubiertos por un estrato arcilloso bastante uniforme en todo el yacimiento que nivela y amortiza un espacio que posteriormente ha sido explotado como pasto. La escasez de estos restos, condiciona la interpretación de unas estructuras probablemente construidas en madera (VigilEscalera, 2003), a partir de un envigado sencillo quizás sobre algún zócalo de piedra sobre el que se habrían levantado las paredes y la cubierta, en su mayoría con materiales de origen vegetal.

Las estructuras de planta cuadrada en este sector, no presentan muchas más evidencias tangibles. Entre ellas, podemos apreciar diferencias entre aquellos fondos rehundidos de planta cuadrada o rectangular totalmente rebajados sobre la superficie de paso de modo parecido a las formas de construcción de las estructuras circulares y, por otro lado, algunas estructuras, entre las que destaca RiI, en donde se aprecia sobre el suelo un rebaje en ángulo recto preparado para constituir el cimiento de un muro de unos $80 \mathrm{~cm}$ de diámetro, que parece constituir el grosor habitual de los zócalos de este tipo de viviendas. Esta estructura parece tener un marcado carácter rupestre a juzgar por la presencia de un gran bloque pétreo caído en donde se advierte la presencia de cinco encajes alineados que podrían haber constituido el soporte para el envigado de un techo. En algunos casos, estas estructuras de planta cuadrada o rectangular aparecen asociadas a pequeños derrumbes de piedras irregulares, por lo que puede suponerse la construcción de paredes algo más sólidas. Las características de los suelos, habitualmente muy rústicos, no difieren en relación con las estructuras circulares.

La interpretación de estos restos es francamente compleja dada la escasez de evidencias. Sin embargo, desplazándonos hacia el nordeste en el prado anejo a la necrópolis, y a unos $125 \mathrm{~m}$ de la iglesia, se ha realizado una cata cuadrangular durante la última intervención y se ha recuperado una potencia de sedimento que alcanza los $50 \mathrm{~cm}$. En este espacio se ha recuperado la esquina de una habitación constituida por dos muros de mampostería irregular que constituyen una de las fases más tardías documentadas en el yacimiento (Fig. 6). El interés de este espacio radica en el hecho de que los niveles más antiguos se han recuperado íntegros pudiendo documentar una intensa utilización del espacio disponible, mediante la identificación del al menos tres fases claramente diferenciadas. La excavación de este sector ha ofrecido numerosas pistas para la interpretación del conjunto, puesto que la sucesión de pavimentos se percibe con claridad. En el suelo de arenisca se documenta un profundo corte en ángulo recto que delimita un espacio cuadrangular de más de $30 \mathrm{~cm}$ de profundidad. Este espacio, inicialmente utilizado en relación con algunas cubetas y tal vez con una estructura circular de fondo realzado (Ci4) es objeto de una primera pavimentación muy tosca para su uso. En un segundo periodo, el corte practicado en la roca es 


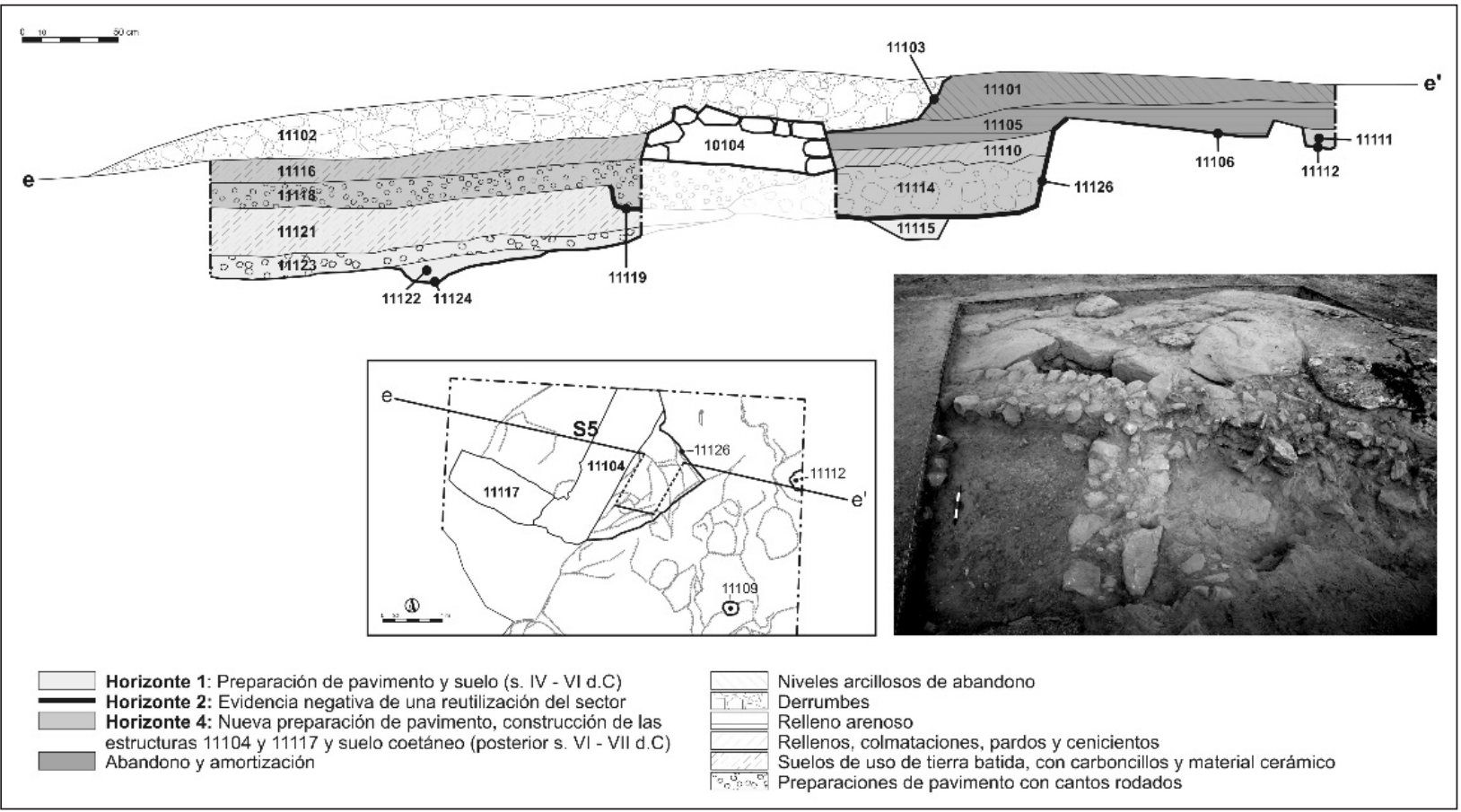

Figura 6: Planta, sección y fotografía de la cata realizada en el área RM4.

utilizado nuevamente, tal vez para usos productivos, en función de las trazas de combustión detectadas en la zona. Nada permite intuir la existencia de un edificio cubierto para esta segunda fase; antes parece que el recinto hubiera sido empleado como un espacio al aire libre que en un momento determinado se amortiza y nivela mediante la deposición de sedimentos de colmatación y derrumbe sobre los cuales se edifica una estructura en piedra cuya esquina hemos conservado (López et al., 2016: I70-I7I).

La presencia de estos espacios productivos, con fuertes trazas de combustión y algunos fragmentos de mena, es habitual en distintos puntos del yacimiento. A día de hoy hemos podido recuperar tres espacios donde se detectan trazas más o menos intensas de una actividad productiva tal vez relacionada con la metalurgia, siendo el más significativo de ellos el relacionado con la floración de suelo rocoso RMi, en relación con las estructuras $\mathrm{CI}_{\mathrm{y}}$ y $\mathrm{R}_{3}$, situadas unos $45 \mathrm{~m}$ al este de la iglesia (Fig. 2). La secuencia estratigráfica de este lugar revela la presencia de un primer momento de utili- zación de una estructura de planta circular como las anteriormente descritas, reutilizada posteriormente como espacio de producción al aire libre y posteriormente amortizada para la construcción de una estructura de planta cuadrada.

En este sector, sobre los niveles de amortización de la estructura $\mathrm{C}_{3}$ se advierte un gran recorte, remoción o rebaje del terreno relleno por los niveles correspondientes a la actividad productiva. Dicho espacio se define en base a la presencia de unidades coetáneas de pequeña entidad que parecen acumulaciones de materiales de distinto tipo: un amontonamiento de arenilla blanquecina, una lechada de argamasa de textura granulosa, una capa de arcilla muy oscura, que tal vez hubiera constituido la base de un hogar y una pequeña acumulación de material arenoso con un remarcable componente calcáreo. En la esquina sudeste, encajado en el ángulo que dibuja el suelo rocoso, se encuentra un hogar delimitado por una estructura de piedra muy arrasada. Este caso no es el único espacio de producción documentado: sobre el suelo de arenisca 
RM2, en un lugar donde no detectamos estructuras de hábitat ni restos de elementos construidos en altura, se aprecia una fortísima rubefacción de un área muy irregular, alrededor de una cubeta trapezoidal (Fig. 5, UE I0238). Las características de este espacio corresponden a grandes rasgos con las anteriormente descritas para RMi, aunque en este caso no detectamos ningún uso posterior tras la amortización del mismo.

\section{DISCUSIÓN}

Los datos presentados refuerzan la idea de que el poblamiento en Revenga es ciertamente dilatado en el tiempo, y se articula en distintas fases que implican una transformación, reconversión y en ocasiones reaprovechamiento de estructuras. La secuencia temporal relativa permite definir la presencia de al menos dos fases de habitación distintas: una de estructuras predominantemente circulares y otra más tardía de estructuras de planta cuadrada. Así lo apunta la estratigrafía de los espacios con mayor acumulación de sedimento, aunque en los casos en donde el suelo rocoso aparece desnudo, la propia disposición de las estructuras permite identificar la misma secuencia cronológica. Si observamos la diferencia de cota entre ellas podemos confirmar que, por regla general, las estructuras de planta cuadrada son posteriores a las de planta circular. La impresión general es que nos encontramos ante una fase inicial del poblamiento que responde a cabañuelas circulares de pequeñas dimensiones más o menos concentradas, y que en algún momento se produce una trasformación del poblado para desarrollar un nuevo tipo de construcciones. Esta segunda fase constructiva, sin embargo, no parece responder a un único periodo, puesto que en determinadas zonas se detecta una superposición de estructuras de planta cuadrada y en otros casos una alternancia entre las estructuras construidas, espacios de trabajo al aire libre y nuevas fases de ocupación posterior.

Los principales interrogantes en el estudio territorial de los asentamientos del Alto Arlanza en general y del yacimiento de Revenga en particular pasan por dilu- cidar la existencia o no de una continuidad o ruptura respecto del mundo antiguo, así como averiguar el origen de la concentración del poblamiento medieval y sus características hasta configurar la red parroquial y aldeana de época feudal. En este sentido, ya se ha apuntado en trabajos anteriores que no existe ninguna prueba que permita plantear la existencia de una continuidad ocupacional en ninguno de los yacimientos antiguos del Alto Arlanza (Padilla y Álvaro, 20IO: 286). Revenga no es una excepción en este sentido, puesto que no se detecta ningún indicio que sugiera la existencia de un poblamiento romanizado anterior al siglo IV. Sin embargo, el registro arqueológico nos permite apuntar hacia la existencia de una ocupación temprana, probablemente en relación con el proceso de cristianización de un territorio de montaña.

En cualquier caso, a juzgar por los materiales cerámicos recuperados y las características generales del asentamiento, la ocupación del enclave es anterior a las cronologías tradicionalmente atribuidas a estos asentamientos, que fechaban su inicio hacia el siglo VIII en el marco de la repoblación llevada a cabo por los reinos cristianos del norte. Los principales volúmenes de cerámica recuperados, por lo general muy parcos, corresponden con una cronología tardoantigua que podría remontarse hasta una horquilla situada entre los siglos IV-VI d. C. (Fig. 7), hecho que corrobora la existencia de estos asentamientos con anterioridad a lo tradicionalmente sugerido.

Las escasas formas recuperadas corresponden a cuencos y platos crateriformes o caliciformes que presentan paralelos en un espacio territorial amplio que comprende la cuenca del Duero y que puede extenderse en función de los casos hasta la cuenca del Ebro y la meseta sur, en los alrededores de Toledo (Larrén et al., 2003: 294). Estas formas abiertas, generalmente con un pie más o menos elevado arraigan en la tradición indígena celtibérica (Burillo et al., 2009: 172-I80) y desaparecen por completo en el periodo altomedieval, siendo prácticamente imposible hallar paralelos similares en época más tardía. Junto con estas formas singulares, se documentan para este periodo temprano, algunos bordes de jarra o redoma que no son en absoluto 
extrańos en el área del Duero (Larrén, et al., 2003: 299), ni tampoco en el territorio que se extiende algo más al sur de la meseta (Caballero, et al., 2003: 244).

Los datos aportados por la arqueología a partir de las intervenciones recientes en este yacimiento sugieren retroceder al menos un par de siglos las cronologías anteriormente manejadas para Revenga (Padilla y Álvaro, 20I0: 293). Las estructuras de hábitat analizadas, así como los espacios abiertos de producción, parecen remontarse como mínimo a los siglos V-VI, en correlación con otras cronologías similares propuestas para enclaves de este tipo (Brogiolo y Chavarría, 2008). De todos modos, una vez las primeras comunidades se hubieron asentado en este espacio, nada parece indicar que se produzca una cesura en la ocupación del territorio del siglo VI en adelante. En otras áreas del tercio norte peninsular el poblamiento parte de una continuidad respecto del mundo bajo imperial, marcada por la evolución y la transformación de las villae, posteriormente abandonadas alrededor de los siglos VIII-IX (Vigil-Escalera, 2007: 245). En cambio, en el caso de Revenga, un poblamiento autóctono instalado en este espacio con anterioridad se mantendrá de manera estable hasta su integración en las estructuras feudales del Alfoz de Lara.

Estas formas alternativas de ocupación del territorio en ningún caso son anómalas en el espacio europeo, que debe ser entendido desde una óptica microregional. En este contexto amplio, nuevas formas de establecimiento rural aparecen en el periodo comprendido entre los siglos IV y V, al margen de la propia evolución de las villae bajoimperiales, que siguen un proceso paralelo de transformación (Schneider 2007). No podemos dejar de lado el hecho de que Revenga se integra en una densa malla de poblamiento en donde se observa una variabilidad de centros y una jerarquización de asentamientos, que en cualquier caso, aparecen siempre relacionados con el edificio de culto.

Esta concentración de asentamientos en el entorno de la Alta Sierra parece cobrar sentido en relación con las rutas trashumantes que se dirigen hacia tierras riojanas a través del valle de Neila. El carácter tradicional de esta ruta, que algunos autores remontan a época pro- tohistórica (Padilla y Álvaro, 20IO: 294) no está reñido con la nueva propuesta cronológica que presentamos. Antes al contrario, permite reforzar la idea de que el poblamiento de la zona se produce en el tránsito entre la antigüedad tardía y la alta edad media en un marco periférico relativamente apartado de los poderes establecidos, a la par que avanza el proceso de cristianización del territorio.

Nos hallamos, pues, ante un proceso de gestación de las estructuras poblacionales de época medieval que arranca en un momento temprano y que experimentará un lento proceso de evolución. Debemos vincular las trasformaciones propias que hemos detectado en el enclave con una adecuación de los espacios a usos dispares relacionados con una actividad ganadera y una agricultura parca y de subsistencia. Estas actividades, fundamentadas en la ganadería y la explotación del bosque parecen ser la característica fundamental del territorio; actividades que, por otro lado, experimentan un cierto auge a lo largo de los siglos V y VI, en un momento de declive de la producción agrícola, siempre en un contexto microregional (Lewit 2009: 79). Los análisis carpológicos y palinológicos en curso, nos permitirán aportar informaciones precisas al respecto que contribuyan a clarificar el panorama interpretativo del asentamiento.

\section{A MODO DE CONCLUSIÓN}

A lo largo de este trabajo hemos descrito e interpretado los nuevos datos que nos ofrece el registro arqueológico, que todavía está en fase de construcción, y en el cual esperamos profundizar a lo largo de los años venideros. Por ello, no parece conveniente presentar aún unas conclusiones cerradas acerca de la interpretación de este registro que permanece en estudio. La investigación acerca del tránsito entre la antigüedad tardía y la alta edad media en el Alto Arlanza, presenta aún hoy más interrogantes que respuestas. Sirvan por tanto estas breves líneas para plantear algunas hipótesis de futuro y para marcar la línea por la que creemos que deben continuar los trabajos de investigación en Revenga. 


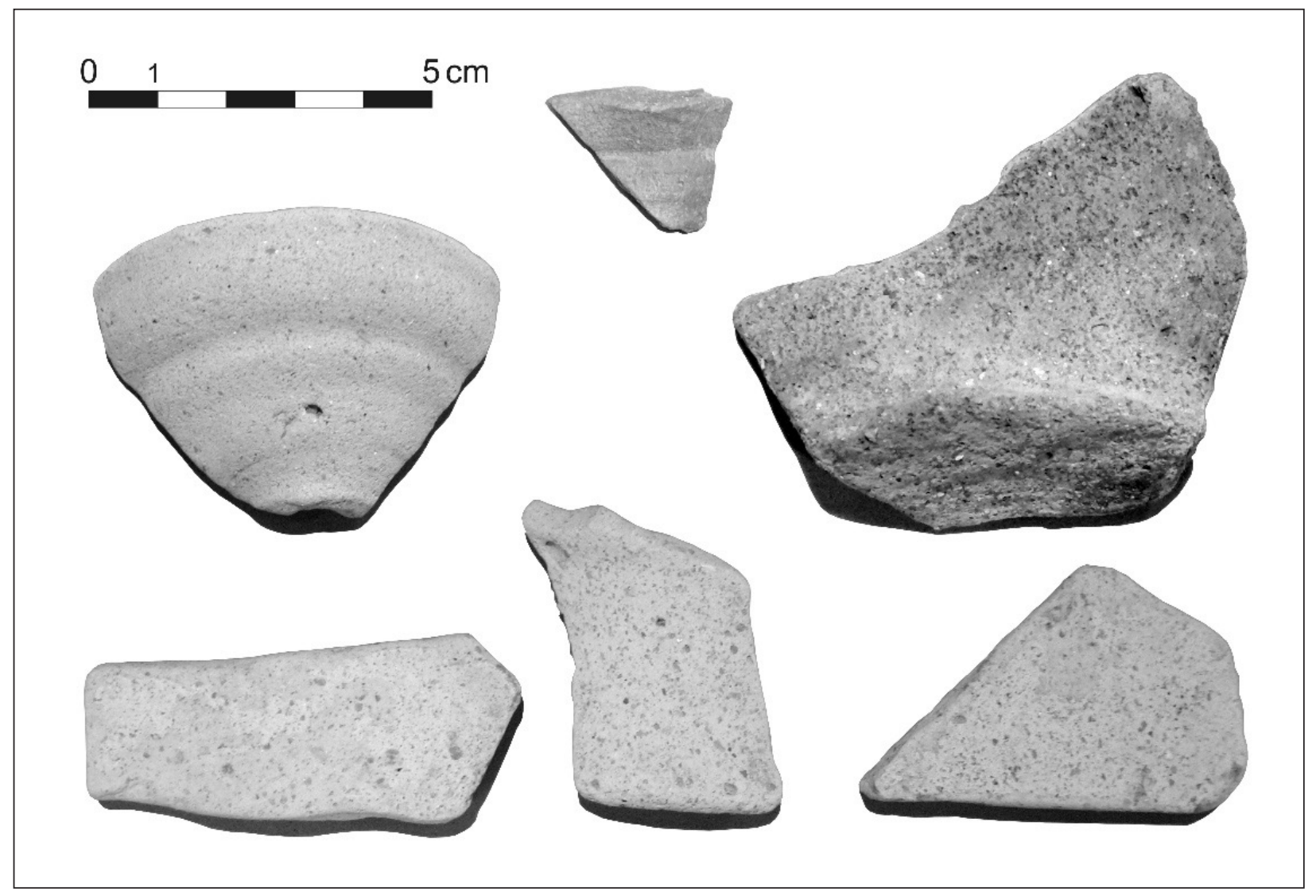

Figura 7: Fotografías de los materiales cerámicos recuperados en Revenga representativos del periodo tardoantiguo, alrededor de los siglos IV - VI.

El yacimiento de Revenga, actualmente en fase de revisión a la luz de las nuevas intervenciones arqueológicas, constituye un núcleo privilegiado para el estudio de los procesos de poblamiento en la zona del Alto Arlanza. Ciertamente, la información disponible sobre la composición interna de estas aldeas es todavía limitada, requiere de un trabajo minucioso de interpretación y debe verse complementado en el futuro por estudios analíticos -fundamentalmente carpológicos de las muestras de sedimento y petrográficos de los materiales cerámicos- y de la excavación de superficies lo más extensas posible. En cualquier caso, hemos superado por primera vez la invisibilidad de las estructuras de hábitat en el conjunto arqueológico del Alto Arlanza, cuyos espacios de poblamiento han quedado habitual- mente constreñidos a la visión fragmentaria que deriva del estudio exclusivo de los conjuntos funerarios.

El necesario cambio de enfoque (Padilla y Álvaro, 20I0: 265) que requiere la aproximación a estas comunidades rurales, pasa por una doble transformación: la primera, evitar la percepción del edificio de culto y el recinto funerario como espacios huérfanos que nada tienen que ver con el poblamiento y, la segunda, construir un registro arqueológico de calidad que pasa por excavar los espacios anexos con la mirada puesta en el espacio habitado, teniendo en cuenta que éste aparece intrínsecamente vinculado con el espacio religioso. En efecto, las características propias de estos espacios habitados que utilizan la superficie rocosa acondicionada o semiexcavada para acoger estructuras de madera son 
comparables a las de otros asentamientos aldeanos tardoantiguos (Álvaro y Padilla, 20I2: 447). Sin embargo, la presencia invariable de un edificio de culto y su respectiva área funeraria en estas aldeas altomedievales contribuye a reforzar el carácter estable y organizado del asentamiento.

En este sentido, queda aún por precisar en el futuro la secuencia de las distintas fases del poblamiento que hemos detectado en Revenga en relación con la propia evolución del espacio sagrado. La intervención de 20I6, centrada en el entorno de la estructura definida como R2 y cuyos datos están todavía en proceso de revisión y estudio, ha permitido conocer algunos de los puntos de contacto entre la necrópolis y el poblado, sugiriendo una presencia de hábitat en este enclave anterior al recinto funerario, al menos en las fases más antiguas, que a medida que avanzan las décadas se estructura y se reordena. Sólo la continuación de los trabajos de campo y el análisis de los restos materiales permitirá corroborar una propuesta interpretativa, que está todavía en construcción.

\section{AGRADECIMIENTOS}

Este trabajo se en incluye entre las tareas habituales del Grupo de Investigación en Arqueología Medieval y Postmedieval de la Universitat de Barcelona (GRAMP. UB), reconocido y consolidado por la Generalitat de Catalunya (20I4 SGR I252). La investigación presentada forma parte de los resultados del proyecto $\mathrm{I}+\mathrm{D}$ titulado «La formación del paisaje medieval: el origen de la red aldeana en el Alto Arlanza» (HAR2or2-33673 MINECO/ FEDER.UE), bajo la dirección de M. D. López. 


\section{BIBLIOGRAFÍA}

Addyman, P. V.; Leigh, D. (1973): «The Anglo-Saxon Village at Chalton, Hampshire: Second Interim Report», Medieval Archaeology, I7, p. I-25. Álvaro, K. (2OI2): El poblamiento altomedieval y sus manifestaciones funerarias en la cuenca del Alto Arlanza (s. IX-X), Barcelona, Universidad de Barcelona. Tesis doctoral en línea (http://hdl.handle.net/10803/8065I).

Álvaro, K.; Padilla, J. I. (20I2): «Arquitectura religiosa y necrópolis rupestres entre el Alto Arlanza y la cabecera del Duero (Ss. IX - XI)», Hortus Artium Medievalium, I6, p. 445-458.

AzkÁrate, A.; Quirós, J. A. (200I): «Arquitectura doméstica altomedieval en la Península Ibérica: Reflexiones a partir de las excavaciones arqueológicas de la Catedral de Santa María de Vitoria-Gasteiz, País Vasco", Archeologia Medievale, 28, p. 25-60.

Brogiolo, G. P.; Chavarría A. (2008): «El final de las villas y las transformaciones del territorio rural en el occidente (siglos V-VIII)», en C. Fernández Ochoa, V. García Entero, F. Gil Sendino (eds.), Villas tardorromanas en Hispania, Gijón, Ediciones Trea, p. 193-2I4.

Burillo, F.; Cano, M. A.; Sáez, M. E. (2009): «La cerámica celtibérica», in D. Bernal y A. Ribera (eds.), Cerámicas hispanorromanas. Un estado de la cuestión, Cádiz, Universidad de Cádiz, p. 19I-187.

Caballero, L.; Retuerce, M.; Sáez, F. (2003): «Las cerámicas del primer momento de Santa María de Melque (Toledo), Construcción, uso y destrucción», in L. Caballero, P. Mateos, M. Retuerce (eds.). Cerámicas tardorromanas y altomedievales en la Península Ibérica. Ruptura y continuidad. II Simposio de Arqueología. Mérida 200I, Madrid, CSIC, p. 225-27I.

Снареlot, J. (1980): «Le fond de cabanne dans l'habitat rural OuestEuropéen: État des questions", Archéologie Médiévale, Io, p. 5-57.

Duval, Y. (1988): Auprès des Sains, corps et âme. L'inhumation «ad sanctos» dans la chrétienté d'Orient et d'Occident du IIIè au VIIè siècle. París, Études Agustiniennes.

Escalona, J. (2002): Sociedad y Territorio en la Alta Edad Media Castellana. La Formación del Alfoz de Lara, Oxford, BARSio79.

García de Cortázar, J. Á. (2012): Historia religiosa del Occidente medieval, Madrid, Akal.

Larrén, H.; Blanco, J. F.; Villanueva, O.; Caballero, J.; Domínguez, A.; Nuño, J.; Sanz, F. J.; Marcos G. J.; Martín, M. Á.; Misiego, J. (2003): «Ensayo de sistematización de la cerámica tardoantigua en la cuenca del Duero", en L. Caballero, P. Mateos y M. Retuerce (eds.). Cerámicas tardorromanas y altomedievales en la Peninsula Ibérica. Ruptura y continuidad. II Simposio de Arqueología. Mérida 200I, Madrid, CSIC, p. 273-306.

LAUWERs, M. (2005): Naissance du cimetière. Lieux sacrés et terre des morts dans l'Occident médiéval, París, Aubier (Collection Historique).

Lauwers, M. (20I3): «De l'incastellamento a l'ineclesiamento», en D. IognaPrat, M. Lauwers, F. Manzel, I. Rosé (dirs.), Cluny, les moines et la société au premier âge féodal. Rennes, Presses Universitaires de Rennes, p. 315-338.

LEWIT, T. (2009): «Pigs, presses and pastoralism: farming in the fifth to sixth centuries AD", Early Medieval Europe, 17, p. 77-91.

López, M. D.; Álvaro, K.; Travé, E. (2016): Yacimiento Arqueológico de Revenga. Iglesia, necrópolis, aldea. Burgos, Diputación de Burgos.

Padilla, J. I.; Álvaro, K. (2008): «El despoblado altomedieval de Cuyacabras (Burgos): Realidad, principios y argumentos», Acta historica et archaeologica mediaevalia, 29, p. 575-604.
Padilla, J. I.; Álvaro, K. (20I0): «Necrópolis rupestres y el poblamiento altomedieval en al Alto Arlanza (Burgos)», En la España Medieval, 33, p. 259-294.

Padilla, J. I.; Álvaro, K. (20IIA): «Los grabados rupestres del despoblado medieval de Revenga (Burgos)», Munibe (Antropologia-Arkeologia), 62, p. 439-459.

Padilla, J. I.; Álvaro, K. (20IiB): «La pileta del despoblado medieval de Revenga (Burgos): a propósito del lavado ritual de los difuntos y sus evidencias arqueológicas", Pyrenae, 42/I, p. 67-100.

Padilla, J. I.; Álvaro, K. (20I3): «Los asentamientos altomedievales del Alto Arlanza (Burgos). El despoblado medieval de Revenga», Pyrenae, 44/I, p. II-4I.

PASTOR, E. (1996): Castilla en el tránsito de la Antigüedad al Feudalismo. Poblamiento, poder politico y estructura social. Del Arlanza al Duero (siglos VII-XI), Valladolid, Junta de Castilla y León.

Quirós, J. A. (2006): «La génesis del paisaje medieval en Álava: la formación de la red aldeana", Arqueología y Territorio Medieval, r3/I, p. 49-94.

Quirós, J. A. (20I4): «Aristocracias, poderes y desigualdad social en la primera edad media en el País Vasco», en R. Catalán, P. Fuentes, J. C. Sastre (eds.), Las fortificaciones en la tardoantigüedad (Élites y articulación del territorio (siglos V - VIII d.C.), Madrid, La Ergástula, p. 143-I58.

Rahtz, P. A. (1976): «Buildings and rural settlements», in D. M. Wilson (ed.), The Archaeology of Anglo-Saxon England, Cambridge, Cambridge University Press, p. 49-98.

Ripoll, G., VelázQuez, I. (1999): «Origen y desarrollo de las parrochiae en la Hispania de la Antigüedad tardía», en P. Pergola (ed.) Alle origini della parrocchia rurale (IV - VII sec.) Ciudad del Vaticano, Sussidi allo Studio delle antichità cristiane, XII.

Ruano, L. (2015): Arqueología de la Arquitectura aplicada a la Protohistoria del Occidente de Asturias (ss. I a. C. - d. C.): el hábitat doméstico en Coaña y San Chuis. Madrid, Universidad Autónoma de Madrid. Trabajo de fin de máster inédito (https://www.researchgate.net/publication/30903373I_ Arqueologia_de_la_Arquitectura_aplicada_a_la_Protohistoria_del_ Occidente_de_Asturias_ss_I_a_C_-_d_C_el_habitat_domestico_ en_Coana_y_San_Chuis)

Schneider, L. (2007): «Structures du Peuplement et formes de l'habitat dans les campagnes du sud-est de la France de l'Antiquité au Moyen Age (IVè - VIIIè s.): Essai de synthèse», Gallia: archéologie de la France antique latine, 64, p. II-56.

Sотомауов, M. (2004): «Las relaciones iglesia urbana-iglesia rural en los concilios hispano-romanos y visigodos», en A. GonZÁLEZ y J. M. Blázquez (eds.), Antigüedad y cristianismo: Monografías históricas sobre la Antigüedad tardía. Sacralidad y Arqueología, Murcia, p. 525-542.

Tejerizo, C. (20I4): Estructuras de fondo rehundido altomedievales en la Península Ibérica, Munibe (Antropologia-Arkeologia), 65, p. $215-237$.

Vigil-EsCAlera, A. (2003): «Arquitectura de tierra, piedra y madera en Madrid (ss. V-IX d.C.). Variables materiales, consideraciones sociales», Arqueología de la Arquitectura, 2, p. 287-29I.

Vigil-Escalera, A. (2007): «Granjas y aldeas altomedievales al norte de Toledo (400-85o d. C)", Archivo Español de Arqueología, 80, p. 239-284.

Wiскнам, C. (2005): Framing the Early Middle Ages. Europe and the Mediterranean, 400-800, Oxford, Oxford University Press.

West, S. E. (1969): «The Anglo-Saxon Village of West Stow: An Interim Report of the Excavations 1965-8", Medieval Archaeology, I3, p. I-20. 
